\title{
A Prospective Randomized Trial Using Four Treatment Modalities for the Treatment of Plantar Fasciitis
}

\author{
by Gerald T. Kuwada, DPM, NMD ${ }^{1 \otimes}$
}

The Foot and Ankle Online Journal 4 (8): 1

Introduction: A prospective randomized study was conducted to determine the efficacy of subjective pain reduction in patients with plantar fasciitis using four treatment modalities.

Methods: One hundred patients (62 females and 38 males) were randomly assigned into four groups $(n=25)$ to receive arch supports, ultrasound treatments, injection or orthotics.

Results: The ultrasound group had the highest average pain reduction of 3.97 after treatment and the highest number of patients who had pain relief after treatment at $81 \%$ or 21/25. Orthotics had the next highest average pain reduction followed by local Marcaine/Triamcinolone injection and arch supports. The orthotic group had more patients who had no pain after using the devices than any of the groups.

Conclusions: No single treatment modality provided complete heel pain relief after treatment. Combining modalities will likely help improve plantar fasciitis than just one modality alone. This study also implies that some patients will require more than just one treatment modality to eliminate the symptoms of plantar fasciitis and that each modality usually decreased heel pain in most patients.

Key words: Heel pain, plantar fasciitis.

Accepted: July, $2011 \quad$ Published: August, 2011

This is an Open Access article distributed under the terms of the Creative Commons Attribution License. It permits unrestricted use, distribution, and reproduction in any medium, provided the original work is properly cited. @The Foot and Ankle Online Journal (www.faoj.org), 2011 All rights reserved.

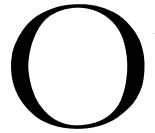

ne of the common foot problems in our society today is plantar fasciitis or plantar fasciopathy. One etiology that appears to be associated with this condition is obesity. Unfortunately, Americans are the heaviest people in the world and the obese American population is increasing at an alarming rate according to the Center of Disease Control (CDC). ${ }^{1,2}$

\footnotetext{
Address correspondence to: Gerald T. Kuwada DPM, NMD. $275 \mathrm{SW} 41^{\text {st }}$ street, Renton, WA 98057.

E-mail: drgeraldkuwada@gwestoffice.net

Office phone: 425-251-9174

Office fax: 425-251-0758

${ }^{1}$ Private practice, $275 \mathrm{SW} 41^{\text {st }}$ street, Renton, WA 98057.
}

The obesity issue is epidemic in America resulting in a myriad of preventable diseases like diabetes, cardiovascular disease, and cancer resulting often in premature death and contributing to the most costliest health care system in the world at plus 2 trillion dollars annually. ${ }^{3}$ The escalating cost of our health care system is a tremendous burden to our society already paying two trillion dollars for the Iraq and Afghanistan wars. Other etiologies for heel pain include trauma, arthritides, overuse injury, foot type, gait abnormalities, masses and tumors. In some cases the etiology is multifactorial. ${ }^{4}$ 
For the obese patient attempting to lose weight by exercise and caloric restriction, having plantar fasciitis makes the situation almost impossible to lose weight. The podiatric physician is faced with a challenging task of determining a viable treatment plan that gets the obese patient exercising with minimal to no pain.

Fortunately, there are many treatment modalities which may be viable for our obese patients as well as others. Ultrasound treatment is non invasive, easy to apply and comfortable for the patient. The high frequency sound stimulates the local tissues and increases the blood flow to the area alleviating inflammation. ${ }^{4}$ Prefabricated and custom orthotics is another treatment recommendation. Studies have shown no significant difference between the orthotics and both have shown to consistently reduce heel pain. ${ }^{4,5}$ Injection therapy is another useful modality that has been used to reduce inflammation in plantar fasciitis. In one study Triamcinolone with Lidocaine proved to be the most effective injection compared to 3 other types of medication and technique types. ${ }^{6}$ For short term treatment low dye strapping has been found to be effective reducing plantar fascial pain.?

As clinicians we observe patients with rigid pes cavus and hyperpronated feet with plantar fasciitis. A study by Zammet and Payne showed that foot orthotics altered rearfoot functioning significantly but couldn't correlate this to symptom reduction for plantar fasciitis patients. ${ }^{8}$ In another study, a combination of treatments was found to reduce heel pain. The study showed that for at least 12 weeks temporary custom orthotics and stretching reduced heel pain in patients. ${ }^{9}$ Medial arch supports reduced heel pain compared to low dye strapping in another study. Both of these treatment modalities were coupled with 9 ultrasound sessions in combination with 3 weeks of calf muscle stretching. ${ }^{10} \quad$ A retrospective study reviewed low dose radiotherapy on 273 patients with plantar fasciitis. They found that there was reduction in heel pain especially in patients 50 years or younger. They could not explain why this occurred but recommended this treatment modality for patients 50 years old or younger in acute cases of plantar fasciitis. ${ }^{11}$
Acupuncture and acupoint PC 7 significantly reduced heel pain compared to acupoint LI 4 which is considered to have analgesic properties. There were 27 patients receiving acupuncture to PC 7 and 25 patients in LI 4 patients. Each patient received 5 treatments for 2 weeks. ${ }^{12}$ Low frequency electrical stimulation was paired with orthotics and stretching versus patients receiving just orthotics and stretching. The results revealed no significant difference between the 2 groups. However, both patient groups had reduction in heel pain after treatments. ${ }^{13}$ Other nonsurgical modalities include the use of radiotherapy, Botox injections, extra-corporeal shockwave therapy, platelet plasma injection and dynamic splinting all demonstrating effective heel pain reduction. ${ }^{14-18}$

This study is unique because it compares one modality against another avoiding a combination of treatments seen in many studies. While combining treatments may be necessary for recalcitrant plantar fasciitis it is unclear which treatment is really reducing heel pain. By isolating each modality, which one will provide the highest pain reduction in patients with plantar fasciitis? This prospective randomized study examines four common treatment modalities: arch support, injection, orthotics and ultrasound for the treatment of plantar fasciitis.

\section{Methods}

There were 100 patients diagnosed with plantar fasciitis randomly selected by the research assistant to receive one of 4 treatment modalities: ultrasound, functional rigid orthotics, over the counter arch supports, and injection. Each participant reviewed and signed consent form regarding this study. There were 25 patients in each treatment group. Each patient rated their pre treatment subjective pain rating based on a $0-10$ scale. Zero equals no pain and 10 is the most severe pain the patient has ever experienced. After one month of treatment the patients pain rating was recorded for every patient in each group. 


\begin{tabular}{|c|c|c|c|c|c|c|}
\hline GROUPS $(n=25)$ & AVG. HT/WT & AVG. AGE & AVG. Pain Reduction & NO PAIN & GRP BMI & $\mathrm{F}: \mathrm{M}$ \\
\hline Orthotics & $1.74 \mathrm{~m} / 94.16 \mathrm{~kg}$. & $50.24 \mathrm{yrs}$. & $2.92 / 10$ & $8 / 25$ & 30.30 & $15: 10$ \\
\hline Ultrasound & $1.69 \mathrm{~m} / 90.73 \mathrm{~kg}$. & $57.50 \mathrm{yrs}$. & $3.95 / 10$ & $5 / 25$ & 30.20 & 14:11 \\
\hline Injection & $1.70 \mathrm{~m} / 97.71 \mathrm{~kg}$. & 55.68 yrs. & $2.74 / 10$ & $2 / 25$ & 32.00 & $15: 10$ \\
\hline Arch Supports & $1.61 \mathrm{~m} / 94.44 \mathrm{~kg}$. & 50.44 yrs. & $2.20 / 10$ & $1 / 25$ & 32.67 & 18:07 \\
\hline
\end{tabular}

Table 1 Treatment group results. Total subjects $(n)=100$, Female to male ratio $=62: 38$. AVG. HT=average height (meters), AVG WT = average weight (kilograms) GRP=group, BMI= Body mass index.

The ultrasound group received 6 treatments of 5 minutes to the painful heel at a cycle of 1.5 over a 2 week period. The arch support group were instructed to purchase an over the counter arch support to fit their feet. The injection group received one injection of $.5 \mathrm{ccs}$ of Triamcinolone and $1 \mathrm{cc}$ of $.5 \%$ plain Marcaine into the painful heel. The orthotic group was casted using the Root, et al., method and a biomechanical examination was also performed on each patient. ${ }^{19}$ The positive casts and biomechanical measurements were sent to a professional orthotic laboratory for fabrication and the orthotic sent to our office for dispensation. The patient's sex, height, weight and age were recorded. The groups BMI or body mass index average was calculated for each group. The average group height, weight and age were also calculated. The average subjective pain rating was calculated. The number of patients with $0 / 10$ pain rating after treatment was recorded for each group. The number of patients whose subjective pain was reduced after treatment in each group was determined. The average pain rating reduction for each group was calculated by the pre-treatment group average pain and subtracted by the post treatment average pain rating for each group.

\section{Results}

There were 62 females and 38 males in this study. The ultrasound group had the highest average pain reduction of 3.97 after treatment and the highest number of patients who had less pain after treatment at $81 \%$ or $21 / 25$.
The ultrasound group also had the lowest BMI average of 30.20. The orthotic group had the second highest subjective pain reduction average after treatment with 2.92. They had the third highest pain reduction patients with $64 \%$ or $17 / 25$. The orthotics group had the highest number of patients who had no pain after treatment at $32 \%$ or $8 / 25$. The ultrasound group had the second highest number with $20 \%$ or $5 / 25$ after treatment. The orthotic group had the second lowest BMI average with 30.3. The injection group had the third highest pain reduction average after treatment with 2.74 . The injection group had the second highest number of patients who improved after treatment with $72 \% 18 / 25$. They had the second highest BMI average with 32. There were $7 \%$ of $2 / 25$ patients who got complete pain relief after injection. The arch support group had the highest average BMI of 32.67. The arch support group had the lowest number of patients with no pain after treatment at $5 \%$ or $1 / 25$. There were $9 / 25$ or $35 \%$ patients who improved after treatment with arch supports. There were 10 patients who weighed over 300 lbs. Eight of 10 were female and the 2 were males. One male weighed $173 \mathrm{~kg}$. (380 lbs.)

\section{Discussion}

Based on the results of this study no one treatment provided complete heel pain relief after treatment. Ultrasound group had the overall highest number of patients who had less pain after treatment at $81 \%$ with injection second at $72 \%$ and orthotic group at $64 \%$. The arch support had the lowest number with $35 \%$. 
Orthotic treatment had the highest number of patients at $32 \%$ who reported no pain after treatment. Whereas, the arch support group had only one patient rate $0 / 10$ pain after treatment. This study implies that some patients will require more than just one treatment modality to eliminate the symptoms of plantar fasciitis. Surprisingly, an over the counter arch support provided some relief in 32\% of the patients in the arch support group. Despite its nonspecificity it was still helpful for some patients. Each of the treatment groups average BMI was over 30 indicating that most of the patients were overweight or obese. This study corroborates weight is an important factor in the etiology and treatment of this common foot diagnosis. In 1980 the author reported on a retrospective study of complete fascial release and overall results and complications. ${ }^{20}$ There were no patients who weighed over $136 \mathrm{~kg}$. (300 lbs.) in that patient population. The female to male ratio was 2:1 as in this study. The overall patient BMI was less than 30 for the patients who participated in the 1980 study. The CDC regards obesity a national health issue. Furthermore, the number one cause of death in women and men is now myocardial infarction linked to obesity. In this study there were 4 times more women than men ( 8 women to 2 men ) that weighed over $136 \mathrm{~kg}$. (300 lbs.) Trying to lose weight by diet and exercise will be difficult for patients with plantar fasciitis. It will be a greater challenge for those who are over $136 \mathrm{~kg}$. (300 lbs.)

Most of the treatments considered in this study are safe and effective in reducing heel pain. However, extra-articular corticosteroid injections has been implicated in adverse reactions including one death from necrotizing fasciitis, injection site cellulitis, atrophy of the fat pad, and tendon rupture. ${ }^{21} \mathrm{Kim}$, et al., reported on a $2.4 \%$ incidence of plantar fascial rupture after receiving an average of 2.67 injections. ${ }^{22}$ The injections consisted of $.5 \mathrm{ccs}$ of dexamethasone plus .5ccs of Triamcinolone, and .5ccs of Xylocaine mixed with $.5 \mathrm{ccs}$ of Marcaine. Thus far, there have been no reported cases of severe complications from Botox $^{\circledR}$ injections and platelet plasma injections.
The author begins treatment for most patients with plantar fasciitis using ultrasound treatment, and arch supports initially. If there is residual heel pain, an injection is added to the treatment regimen. If there is still recalcitrant heel pain, orthotics is recommended. In about $87 \%$ of the patients diagnosed with plantar fasciitis there is heel pain reduction. Thirteen percent don't improve or worsen after exhaustive conservative treatment and $90 \%$ of these patients proceed with complete fascial release with a 98\% success rate. This is over 35 years of clinical experience and observation.

This study is unique because only one treatment modality was administered to each patient in their respective groups. Many of the studies used multiple treatment modalities with the treatment being investigated to determine if there was significant pain reduction to plantar fasciitis patients. The combining of treatments may show pain reduction but we cannot ascertain which treatment helped more than the others. The authors study clearly demonstrated that each modality decreased heel pain usually in most patients.

\section{References}

1. Flegal KM, Carroll MD, Ogden CL, Curtin LR. Prevalence and trends in obesity among U.S adults 1999-2008. JAMA 2010 303: 235-241.

2. CDC REPORT for 2010. National Center for Chronic Disease Prevention and Health Promotion, CDC: Freedman, D.S on Obesity 2010, 73-78.

3. WHO Report 2010. WHO 2010 Geneva, Switzerland.

4. Healey K. Chen K. Plantar fasciitis: current diagnostic modalities and treatments. Clin Pod Med Surg 2010 7(3): 396380 .

5. Landorf KB, Keenan AM, Herbert RD. Effectiveness of different types of foot orthoses for the treatment of plantar fasciitis. JAPMA 2004 94: 542-549.

6. Baldassin V, Gomes CR, Beraldo PS. Effectiveness of prefabricated and customized foot orthoses made from low cost foam for noncomplicated plantar fasciitis: a randomized controlled trial. Arch Phys Med Rehab 2009 90: 701-706. 
7. Kalaci A, Cakici H, Hapa O, Yanat N, Dogamaci Y, Sevinc TT. Treatment of plantar fasciitis using four different local injection modalities: A randomized, prospective clinical trial. JAPMA 2009 99: 108-113.

8. Zammet GV. Payne CB. Relationship between positive clinical outcomes of foot orthotic treatment and changes in rearfoot kinematics. JAPMA 2007 97: 207-212.

9. Drake M, Bittenbender G, Boyles RE. The short term effects of treating plantar fasciitis with a temporary custom foot orthosis and stretching. J Orthop Sports Phys Ther 2011 41: 221-231.

10. El Salam MS, Elhafz YN. Low-dye taping versus medial arch support in managing pain and pain related disability in patients with plantar fasciitis. Foot Ankle Spec 2011 4: 86-91. 11. Osborne HR, Allison GT, Hanna C. Treatment of plantar fasciitis by low dye taping an iontophoresis: Short term results of a double blinded, randomized placebo controlled clinical train of Dexamethasone and Acetic acid. BJSM 2006 40: 545-549. 12. Zhang SP, Yip TP, Li QS. Acupuncture treatment for plantar fasciitis: A randomized controlled trial with six months follow-up. Evid Based Complement Alternat Med 2009: 23. 13. Stratton M, McPoil TG, Cornwall MW, Patrick K. Use of low frequency electrical stimulation for the treatment of plantar fasciitis. JAPMA 2009 99: 481-488.

14. Hajtmanova E, Kinclova I, Kostkova L, Hajtman A, Pec M. Low dose radiotherapy in the treatment of plantar fasciitis. Klin Onkol 2010 23: 104-110.

15. Rawicki B, Sheean G, Fung VS, Goldsmith S, Morgan C, Novak I. Cerebral Palsy Institute. Botulinum toxin assessment, intervention and aftercare for pediatric and adult niche indications including pain: international consensus statement. Eur J Neurol 2010 Suppl 2: 122-134.

16. Metzner G, Dohnalek C, Agner E. High energy extracorporeal Shock Wave Therapy for the Treatment of Chronic plantar fasciitis. Foot Ankle Int 2010 31: 790-796. 17. Peerbooms JC, van Laar W, Faber F, Schuller HM, van der Hoeven $\mathrm{H}$, Gosens T. Use of platelet rich plasma to treat plantar fasciitis: design of multi centre randomized controlled trial. BMC Musculoskeletal Disorders 2010 13:11: 69.

18. Sheridan L, Lopez A, Perez A, John MM, Willis FB, Shanmugam R. Plantar fasciopathy treated with dynamic splinting. A randomized controlled trial. JAPMA 2010 10:161165.

19. Root ML, Orien WP, Weed JH. Normal and Abnormal Function of the Foot. Clinical Biomechanics Corp Los Angeles, 1977.

20. Kuwada GT, Gormley J. Retrospective ananlysis of calcaneal spur removal and complete fascial release for the treatment of chronic heel pain. JFS 1992 11: 166-169.

21. Brinks A, Koes BW, Volkers AC, Verhaar JA, BiemaZeinstra SM. Adverse effects of extra-articular corticosteroid injections: a systemic review. BMC Musculoskeletal Disorders 2010 11:206.

22. Kim C, Cashollar MR, Mendicino RW, Catanzariti AR, Fuge L. Incidence of plantar fascia ruptures following corticosteroid injection. Foot Ankle Spec 2010 3: 335-337. 